\title{
A FULL AND FAITHFUL NERVE FOR 2-CATEGORIES
}

\author{
M. BULLEJOS, E. FARO AND V. BLANCO
}

\begin{abstract}
The notion of geometric nerve of a 2-category (Street, 18) provides a full and faithful functor if regarded as defined on the category of 2-categories and lax 2-functors. Furthermore, lax 2-natural transformations between lax 2-functors give rise to homotopies between the corresponding simplicial maps. These facts allow us to prove a representation theorem of the general non abelian cohomology of groupoids (classifying non abelian extensions of groupoids) by means of homotopy classes of simplicial maps.
\end{abstract}

\section{INTRODUCTION}

The work presented here began as a search for a definition of nerve of a 2category which could be used to prove a representation theorem for the non abelian cohomology of groupoids. This cohomology, which generalizes the non abelian cohomology of groups given by Dedecker [7], is defined in [2] so as to classify non abelian extensions of groupoids by "Schreier invariants" in a way that generalizes the classical Schreier theory of groups, (see 16 and 10. It was clear that in order to obtain the desired representation in terms of homotopy clases of simplicial maps, a nerve functor which was full and faithful was needed. Unfortunately this property was not satisfied by any of the various notions of nerve of a 2-category existing in the literature. It was only after establishing Definition 3.1 that the authors realized that this definition of nerve is essentially the same as that of nerve of a bicategory of Duskin (see 8] ) and that it agrees on objects with Street's definition of nerve of a 2-category (the so called geometric nerve, [18]).

It is a fairly intuitive fact that, as observed by Grothendieck in the early 1960's, any (small) category gives rise to a simplicial set (called the nerve of the category) and that functors give rise to simplicial maps between the corresponding nerves. A functor "nerve" is thus obtained from the category Cat of small categories and functors to that of simplicial sets, and the fact that this functor is full and faithful has important consequences such as the fact that Cat has a model structure whose homotopy category is equivalent to that of simplicial sets.

Going up one dimension, a similar notion of "nerve of a 2-category", associating with each 2-category a simplicial set which captures the essential aspects of the 2 -dimensionality of the structure of the 2-category, becomes useful and necessary in a variety of contexts ([3], 8], [14, [18). In this case, however, several notions of nerve have been proposed, each with its own applications, but none of them providing a full and faithful functor.

In this paper we show that if one considers lax 2-functors as the arrows between 2-categories then, on the one hand, Street's notion of nerve of a 2-category can be given a simple and natural definition, and, on the other hand, the resulting nerve functor is defined on all lax 2-functors, that is, it becomes a functor on the category 
2-Cat lax $_{\text {an }}$ of small 2-categories and lax 2-functors. As a result this nerve functor is full and faithful, allowing us to prove the desired representation theorem.

The plan of the paper is as follows: Section 2 is an overview of a general approach to the definition of nerve of the objects of a category. In Section 3 we review three different notions of nerve of a 2-category and see them as fitting the general scheme indicated in Section 2 Special attention is given to the nerve defined by Street 18 and the fact that it coincides on objects with that of our Definition 3.1 Section 4 is devoted to prove that the geometric nerve of Definition 3.1 is full and faithful (Proposition 4.3) and that to deformations of lax 2-functors there correspond homotopies between their associated simplicial maps. These results are crucial to establish the representation theorem of non abelian cohomology of groupoids in Section 5 .

To end this introduction we indicate that all lax 2-functors considered in this paper will be normal ${ }^{1}$. Thus, the expression "lax 2 -functor" will be used without further notice to mean "normal lax 2-functor", that is, our convention is that "a lax 2-functor takes identity 1-cells to identity 1-cells, the structural natural transformation for identities is an identity, and the structural natural transformation for composition, $\sigma$, satisfies the additional requirement that for any 1-cell $f: A \rightarrow B$,

$$
\sigma_{1_{A} f}=\sigma_{f 1_{B}}=\mathrm{id}_{\bar{f}}
$$

(where $\operatorname{id}_{\bar{f}}$ is the identity 2-cell of the image $\bar{f}$ of $f$ )."

\section{General Definition of nerve}

The classical definition of nerve of a category reflects the fact that $\Delta$, the simplicial category, whose objects are those particular posets that are the finite non-empty linear orders, $\mathbf{1}=[0]=\{0\}, \mathbf{2}=[1]=\{0 \leq 1\}, \ldots$ (and whose arrows are all functors or monotonic maps between them), can be regarded as a full subcategory of Cat. Then, given that the $n$-simplices of a category $\mathcal{C}$ are just the functors $[n] \rightarrow \mathcal{C}$, the fact that the nerve functor is full and faithful just reflects the fact that $\Delta$ is an adequate $^{2}$ subcategory of Cat.

By general categorical arguments, given a functor i : $\Delta \rightarrow \mathcal{A}$, one can define the $n$-simplices of an object $A$ of $\mathcal{A}$ as the arrows from $\mathbf{i}([n])$ to $A$, that is, one can define

$$
\operatorname{Ner}(A)_{n}=\mathcal{A}(\mathbf{i}([n]), A) .
$$

The functoriality of $\mathbf{i}$ provides face and degeneracy operators satisfying the simplicial identities so that $\operatorname{Ner}(A)$ becomes a simplicial set. In this way one obtains a functor

$$
\text { Ner }: \mathcal{A} \rightarrow \text { Set }^{\Delta^{\mathrm{op}}}=\text { Sset }
$$

defined on objects as

$$
\operatorname{Ner}(A)=\mathcal{A}(\mathbf{i}(-), A)
$$

and on arrows $f: A \rightarrow B$ in $\mathcal{A}$ via composition: $\operatorname{Ner}(f)=\bar{f}$ is defined as the simplicial map

$$
\operatorname{Ner}(A)_{n} \stackrel{\bar{f}_{n}}{\longrightarrow} \operatorname{Ner}(B)_{n}, \quad \bar{f}_{n}(\alpha)=f \circ \alpha .
$$

\footnotetext{
${ }^{1}$ Here "normal" means "strictly identity preserving."

${ }^{2}$ The concept of an adequate subcategory was introduced by Isbell in [9].
} 
This construction provides a sensible nerve of the objects of $\mathcal{A}$ if the resulting functor (2) is full and faithful (i.e., if $\Delta$ is adequate in $\mathcal{A}$ ). If it is not, it may still be possible to define a sensible nerve of the objects of $\mathcal{A}$ if $\Delta$ is adequate in some category $\mathcal{A}^{*}$ that "enlarges" $\mathcal{A}$ in the sense that there is a faithful inclusion $\mathcal{A} \hookrightarrow \mathcal{A}^{*}$. In that case, the nerve of an object $A \in \mathcal{A}$ can be defined as the nerve of $A$ as an object of $\mathcal{A}^{*}$. Most of the definitions of nerves that are found in the literature fit this general scheme. For example, the definitions of nerve of a groupoid and nerve of a 2-groupoid found in 14 fit this pattern, as they also do all the three definitions of nerve of a 2-category that we review in Section 3

\section{THREE NERVE FUNCTORS FOR 2-CATEGORIES}

Street in [18] defines a functor $\mathcal{O}: \Delta \rightarrow \omega$-Cat to the category of (strict) $\omega$ categories and strict functors. Then, he defines the nerve of an $\omega$-category by means of this embedding, following the general scheme described above. On the other hand, there is a canonical functor $\omega$-Cat $\rightarrow 2$-Cat which kills all non identity cells at dimensions higher than 2 and by composing it with $\mathcal{O}$ an embedding $\overline{\mathcal{O}}: \Delta \rightarrow 2$-Cat results such that for any simplex $[n] \in \Delta$ the 2-category $\overline{\mathcal{O}}_{n}=\overline{\mathcal{O}}([n])$ is the free 2 -category in the simplex $[n]$. The resulting nerve functor $\mathrm{Ner}_{\overline{\mathcal{O}}}: 2$-Cat $\rightarrow$ Sset is not full, but the embedding $\overline{\mathcal{O}}$ has the following universal property: for any 2 category $\mathbf{A}$ the set of strict 2-functors $\overline{\mathcal{O}}_{n} \rightarrow \mathbf{A}$ (or $n$-simplices of $\mathbf{A}$ ) is in natural and bijective correspondence with the set of lax 2-functors $[n] \rightarrow \mathbf{A}$. Due to this universal property, the simplicial set $\operatorname{Ner}_{\overline{\mathcal{O}}}(\mathbf{A})$ can also be defined in the following way:

Definition 3.1 (The geometric nerve). Consider the full and faithful functor

$$
\text { i : } \Delta \rightarrow 2 \text {-Cat } \text { lax }_{\text {la }}
$$

defined by regarding each simplex $[n]$ as a 2-category whose only deformations are the required identities (this forces all lax 2-functors $[n] \rightarrow[m]$ to be just functors or monotonic maps). Using this embedding as in (3) one gets a nerve functor

$$
\text { Ner : 2-Cat } \text { lax }_{\rightarrow} \rightarrow \text { Sset }
$$

such that for any 2-category $\mathbf{A}, \operatorname{Ner}(\mathbf{A})$ is the simplicial set

$$
\operatorname{Ner}(\mathbf{A})=\operatorname{LaxFun}(\mathbf{i}(-), \mathbf{A})
$$

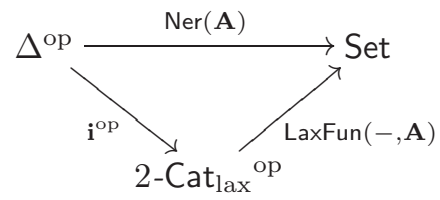

By the above universal property, we obtain a commutative diagram

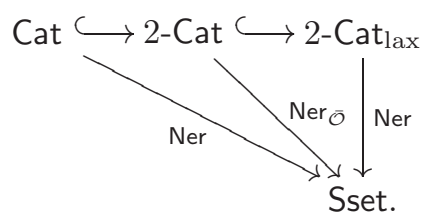

In other words, the geometric nerve functor just defined on 2-categories agrees with the usual nerve of categories and with Street's nerve of 2-categories. Furthermore, if the geometric nerve is defined for 2-groupoids and that it agrees with the definition of nerve of a 2-groupoid given by Moerdijk-Svensson in [14. It should be noted also 
that the definition of the geometric nerve agrees also with the definition of nerve of a bicategory given by Duskin ([8], p. 238).

Two other useful definitions of nerves of 2-categories can be deduced from an embedding, $\mathbf{N}:$ 2-Cat $\hookrightarrow$ BSset, of 2-Cat into the category of bisimplicial sets, $\mathrm{BS} s e t=\mathrm{Set}^{\Delta^{\mathrm{op}} \times \Delta^{\mathrm{op}}}$ (see diagram (5) below), and from two corresponding notions of nerve of a bisimplicial set. The embedding $\mathbf{N}$ of 2-Cat into bisimplicial sets is obtained from the usual nerve of categories by means of which one can regard a 2-category as a bisimplicial set in the following way. Given a 2-category $\mathbf{A}$ let us write $A_{0}, A_{1}$ and $A_{2}$ for the sets of zero-, one- and two-cells of $\mathbf{A}$. Then, we can identify the 2-category $\mathbf{A}$ with the bisimplicial set $\mathbf{N}(\mathbf{A})$ given by the diagram

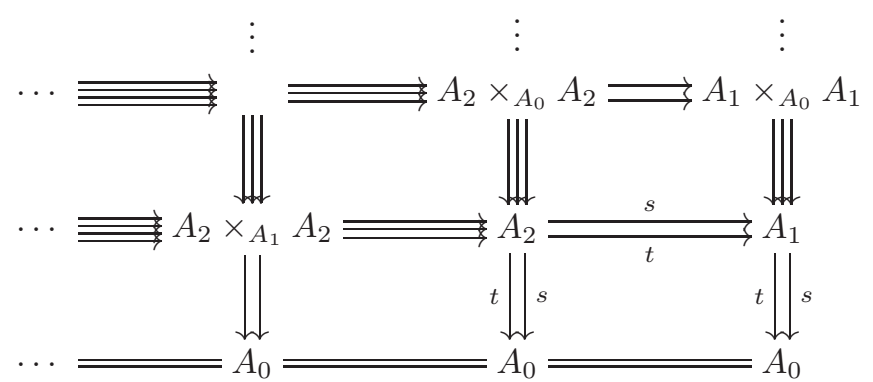

whose arrows and columns represent nerves of categories all of which are determined by $\mathbf{A}$. This construction gives an embedding $\mathbf{N}$ : 2-Cat $\hookrightarrow$ BSset which can be regarded as a sort of "bi-nerve" or "double nerve" of 2-categories.

This embedding can be composed with two different "nerve functors" of bisimplicial sets. The first of them is the Artin-Mazur codiagonal (see [6]) of a bisimplicial set, $\bar{W}:$ BSset $\rightarrow$ Sset, right adjoint to the total dec functor Tdec : Sset $\rightarrow$ BSset, in turn, induced by the ordinal sum functor $+_{\text {or }}: \Delta \times \Delta \rightarrow \Delta$. Note that $\bar{W}$ can be defined by the general procedure for defining nerves given above (see (3)), if one uses the embedding of $\Delta$ into BSset obtained by composing the total dec functor with the Yoneda embedding of $\Delta$, Tdec $\circ \mathbf{y}: \Delta \hookrightarrow$ BSset. The composite $\bar{W} \circ \mathbf{N}$ is a "nerve" of 2-categories one of which applications can be seen in in [3].

The second "nerve functor" of bisimplicial sets comes, again by the general procedure (3), from the composition $\delta^{*} \mathbf{y}$ of the Yoneda embedding of $\Delta$ with the left adjoint $\delta^{*}:$ Sset $\rightarrow$ BSset to the functor induced by the diagonal $\delta: \Delta \rightarrow$ $\Delta \times \Delta$, so that we have a further nerve on bisimplicial sets and on 2-categories $\operatorname{Ner}_{\delta}:$ 2-Cat $\rightarrow$ Sset. In this case the nerve of a 2-category $\mathbf{A}$ obtained as the composite $\operatorname{Ner}_{\delta} \circ \mathbf{N}$ is the diagonal of the bisimplicial set $\mathbf{N}(\mathbf{A})$.

Each of the three nerves of 2-categories has its own interest in homotopy theory. In [4] and [5] it has been proved that the three nerves of a 2-category are homotopically equivalent. But only the geometric nerve can be extended to lax 2-functors so that the corresponding nerve on $2-\mathrm{Cat}_{\mathrm{lax}}$ is full and faithful.

\section{The FUlL AND FAITHFul GEOMETRIC NERVE FUNCTOR}

In the following proposition we spell out our definition of the geometric nerve.

Proposition 4.1. Let $\mathbf{A}$ be a 2-category, the geometric nerve $\operatorname{Ner}(\mathbf{A})$ is the following simplicial set:

- Its vertices are the objects of $\mathbf{A}$, 
- 1-simplices are the arrows $A_{0} \stackrel{f}{\rightarrow} A_{1}$ of $\mathbf{A}$, with faces

$$
d_{0}(f)=A_{1} \quad \text { and } \quad d_{1}(f)=A_{0},
$$

- 2-simplices are the diagrams $\Delta=(g, h, f ; \alpha)$ of the form

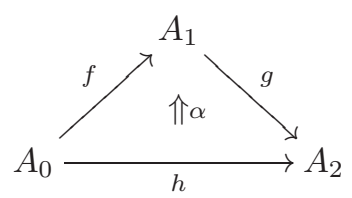

with $\alpha: h \rightarrow g f$ a 2-cell in $\mathbf{A}$, and faces which are the 1-simplices opposite to the indicated vertex, that is,

$$
d_{0}(\Delta)=g, \quad d_{1}(\Delta)=h, \quad \text { and } \quad d_{2}(\Delta)=f
$$

- 3-simplices are "commutative" tetrahedral $\Theta$ of the form

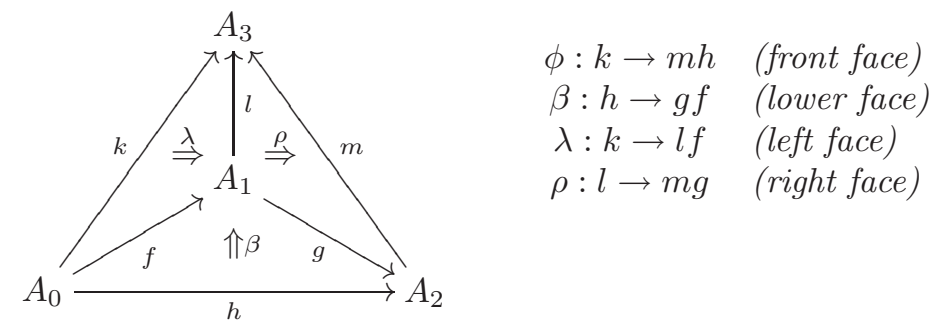

where by commutativity of the tetrahedron we mean that the following square of 2-cells commutes

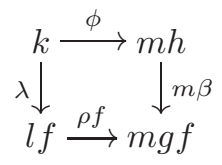

The face operators for such tetrahedron are, as in the case of a 2-simplex, the 2-simplices opposite to the vertex indicated by the operator,

$$
\begin{aligned}
& d_{0}(\Theta)=\Delta_{0} \text { is the right face }(\rho), \\
& d_{1}(\Theta)=\Delta_{1} \text { is the front face }(\phi), \\
& d_{2}(\Theta)=\Delta_{2} \text { is the left face }(\lambda), \text { and } \\
& d_{3}(\Theta)=\Delta_{3} \text { is the lower face }(\beta) .
\end{aligned}
$$

- At higher dimensions $\operatorname{Ner}(\mathbf{A})$ is coskeletal.

It is also useful to have an explicit description of the simplicial map that the nerve functor associates to a lax functor.

Proposition 4.2. Let $F: \mathbf{A} \rightarrow \mathbf{B}$ be a lax functor between 2-categories, with structure map $\sigma$. Then $\bar{F}=\operatorname{Ner}(F): \operatorname{Ner}(\mathbf{A}) \rightarrow \operatorname{Ner}(\mathbf{B})$ is the simplicial map given by:

for 0-simplices $A \in \operatorname{Ner}(\mathbf{A})_{0}, \bar{F}_{0}(A)=F(A)$,

for 1-simplices $f \in \operatorname{Ner}(\mathbf{A})_{1}, \bar{F}_{1}(f)=F(f)$, 
for 2-simplices $\Delta \in \operatorname{Ner}(\mathbf{A})_{2}$, as above, $\bar{F}_{2}(\Delta)$ is obtained from the diagram

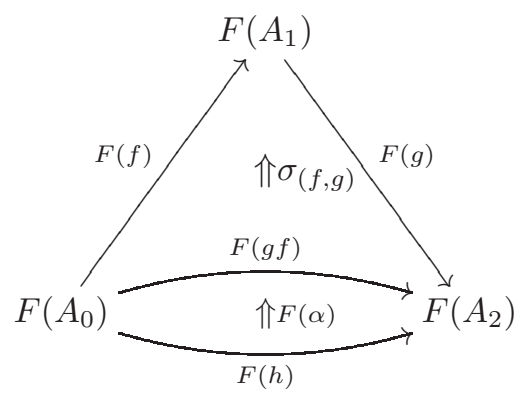

by the vertical composition of deformations: $\sigma_{(f, g)} F(\alpha)$, That is

$$
\bar{F}_{2}(g, h, f ; \alpha)=\left(F(g), F(h), F(f) ; \sigma_{(f, g)} F(\alpha)\right) .
$$

At higher dimensions $\operatorname{Ner}(F)$ is defined in the unique possible way, using the fact that in dimension 3 and above any simplex is determined by its faces.

Next proposition shows that the construction of the geometric nerve functor is full and faithful.

Proposition 4.3. The geometric nerve functor Ner : 2-Cat lax $_{\rightarrow} \rightarrow$ Sset is full and faithful.

Proof. We first prove that Ner is full. Let $\Phi: \operatorname{Ner}(\mathbf{A}) \rightarrow \operatorname{Ner}(\mathbf{B})$ be a simplicial map, then we define a lax functor $F: \mathbf{A} \rightarrow \mathbf{B}$ acting on objects as the vertex part of $\Phi: F(A)=\Phi_{0}(A)$, on arrows as the edges part of $\Phi: F(f)=\Phi_{1}(f)$, and on a 2 -cell $\alpha: h \rightarrow f$ as the 2-cells part of $\Phi$ acting on the following 2 -simplex in $\operatorname{Ner}(\mathbf{A})$

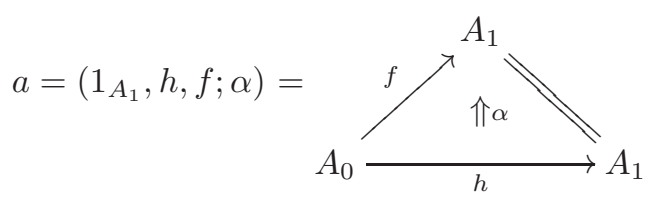

$F(\alpha)=\Phi_{2}(a)$. To complete the definition of $F$ we define its structure map: given a composable pair of arrows $A_{0} \stackrel{f}{\rightarrow} A_{1} \stackrel{g}{\rightarrow} A_{2}$ in $\mathbf{A}$, we consider the 2-simplex in $\operatorname{Ner}(\mathbf{A})$ given by the diagram

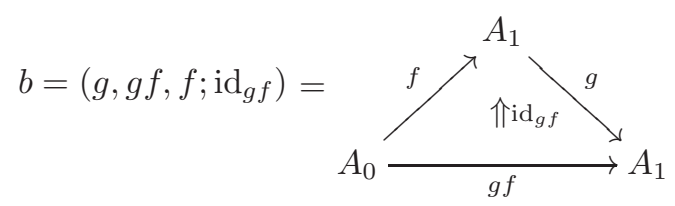

then the structure map $\sigma$ is defined by saying that $\sigma_{(f, g)}^{A_{0} A_{1} A_{2}}$ is the 2-cell that is the interior of the 2-simplex $\Phi_{2}(b)$.

To see that the nerve functor is faithful we first observe that for any lax functor $F: \mathbf{A} \rightarrow \mathbf{B}$ and any 2-cell $\alpha: h \rightarrow f$ in $\mathbf{A}$, if $a=\left(1_{A_{1}}, h, f ; \alpha\right)$ is the 2-simplex in 
$\operatorname{Ner}(\mathbf{A})$ indicated above, then $\operatorname{Ner}(F)_{2}(a)$ is the 2-simplex given by the diagram

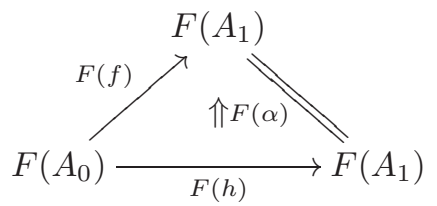

We also observe that, for any composable pair $(f, g)$ in $\mathbf{A}$, the structure morphism $\sigma_{(f, g)}^{A_{0} A_{1} A_{2}}$ is the 2-cell that is the interior of the 2 -simplex $\operatorname{Ner}(F)_{2}(b)$, where $b$ is as above. Therefore two lax 2-functors which have the same nerve coincide on objects, arrows and 2-cells and also have the same structure map.

The nerve construction not only relates lax 2-functors with simplicial maps but it also relates lax 2-natural transformations with homotopies. In the next proposition, whose proof we leave to the reader, we state that lax 2-natural transformations between lax 2-functors give rise to homotopies between their nerves and that these homotopies satisfy a special property. Remember that for $n \geq 3$ the $n$-simplices of a 2-category are completely determined by their boundary (the faces) and therefore a homotopy to the nerve of a 2 -category is completely determined by its 2 -trucation.

Proposition 4.4. Let F, $G: \mathbf{A} \rightarrow \mathbf{B}$ be two lax 2-functors between 2-categories. Every lax 2-natural transformation $\alpha: F \rightarrow G$ induces a homotopy $h=\operatorname{Ner}(\alpha)$ : $\operatorname{Ner}(G) \rightarrow \operatorname{Ner}(F)$ such that the image by $h_{1}^{1}: \operatorname{Ner}_{1}(\mathbf{A}) \rightarrow \operatorname{Ner}_{2}(\mathbf{B})$ of any 1-simplex in $\operatorname{Ner}(\mathbf{A})$ is a 2-simplex which is completely determined by its boundary.

Proposition 4.5. Let $\mathbf{A}$ be a 2-category and $\mathbf{B}$ a 2-groupoid. If $F, G: \mathbf{A} \rightarrow \mathbf{B}$ are two lax 2-functors, there exists a homotopy from the simplicial map $\bar{F}=\operatorname{Ner}(F)$ to $\bar{G}=\operatorname{Ner}(G)$ if and only if there is a lax 2-natural transformation $\alpha: G \rightarrow F$.

Proof. One implication is a consequence of Proposition 4.4 To prove the converse, let $h: \bar{F} \rightarrow \bar{G}$ be a homotopy. Then, for any object $A$ in $\mathbf{A}, h_{0}^{0}(A): G(A) \rightarrow F(A)$ since $d_{0}\left(h_{0}^{0}\right)=F$ and $d_{1}\left(h_{0}^{0}\right)=G$. We define a lax 2-natural transformation $\alpha$ by defining its $A$-component as $\alpha_{A}=h_{0}^{0}(A)$. In order to define the structure morphism, let now $f: A \rightarrow B$ be a 1-cell in $\mathbf{A}$. The homotopy identities imply that we have the following 2-cells of $\mathbf{B}: h_{0}^{1}(f): d \rightarrow F(f) \alpha_{A}$ and $h_{1}^{1}(f): d \rightarrow \alpha_{B} G(f)$ with $d=d_{1} h_{0}^{1}(A)=d_{1} h_{1}^{1}(A): G(A) \rightarrow F(B)$
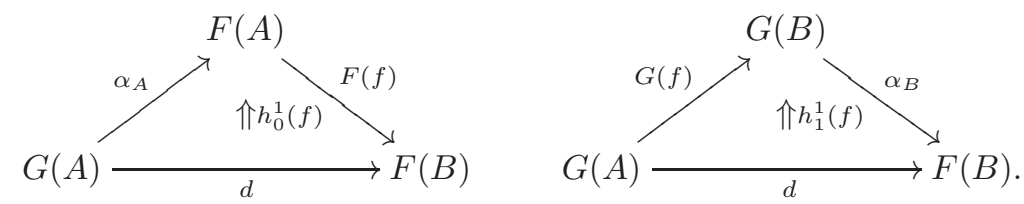

Since $\mathbf{B}$ is a groupoid, the 2-cell $h_{1}^{1}(f)$ is invertible and we can define the structure map $s(f)=h_{0}^{1}(f)\left(h_{1}^{1}(f)\right)^{-1}$. The homotopy identities in the next dimension guarantee the coherence condition so that $(\alpha, s)$ is a lax 2-natural transformation from $G$ to $F$.

\section{REPRESENTATION THEOREM FOR NON ABELIAN COHOMOLOGY OF GROUPOIDS}

As an immediate application of the full and faithful geometric nerve of 2-categories, we give a representation theorem for the 2-dimensional non abelian cohomology of groupoids defined in 2, in terms of homotopy classes of simplicial maps. 
Given two groups, $G$ and $K$, the ("non abelian") extensions of $G$ with kernel $K$ were classified by Dedecker by means of a two dimensional non abelian cohomology, $H_{\text {Aut }(K)}^{2}(G, K)$. This is a set of cohomology classes of 2-cocycles defined by taking coefficients in the crossed module $K \rightarrow \operatorname{Aut}(K)$ of automorphisms of $K$. It is proved in 2 that, by regarding the group(oid) $G$ as a (2-discrete) 2-groupoid, and by taking into account the equivalence between the category of crossed modules and that of 2-groupoids, this cohomology can be represented in terms of lax 2-functors and in fact one has

$$
H_{\text {Aut }(K)}^{2}(G, K)=\operatorname{LaxFun}[G, \operatorname{AUT}(K)]
$$

where $\operatorname{AUT}(K)$ is the 2-groupoid associated to the crossed module $K \rightarrow \operatorname{Aut}(K)$ and LaxFun $[G, \operatorname{AUT}(K)]$ denotes the set of connected components of the category $\operatorname{LaxFun}(G, \operatorname{AUT}(K))$ of lax 2-functors and lax 2-natural transformations from $G$ to $\operatorname{AUT}(K)$. This identification of 2-cocycles with lax 2-functors follows immediately from the observation that Dedecker's definition of cocycle and cocycle condition are exactly the definition of a lax 2-functor and coherence of a lax 2-functor. On this basis 2] defines the 2-cohomology of a groupoid $\mathcal{G}$ with coefficients in a family of groups $\mathcal{K}=\left\{K_{x}\right\}_{x \in \mathcal{G}}$ indexed by the objects of $\mathcal{G}$ as the set

$$
H_{\text {Aut }(\mathcal{K})}^{2}(\mathcal{G}, \mathcal{K})=\operatorname{LaxFun}_{*}[\mathcal{G}, \operatorname{AUT}(\mathcal{K})]
$$

of connected components of lax 2-functors which are the identity on objects. This cohomology, which obviously generalizes Dedecker's, can also be given in terms of cohomology classes of cocycles and it classifies general non abelian extensions of a groupoid $\mathcal{G}$ by the family of groups $\mathcal{K}$ indexed by the objects of $\mathcal{G}$. On the other hand, Propositions 4.3 and 4.5 above give, as an immediate corollary, the following representation theorem of non abelian cohomology in terms of homotopy classes of simplicial maps:

Theorem 5.1. Given a groupoid $\mathcal{G}$ and any family $\mathcal{K}$ of groups indexed by the objects of $\mathcal{G}$, there is a bijection

$$
H_{\text {Aut }(\mathcal{K})}^{2}(\mathcal{G}, \mathcal{K}) \cong[\operatorname{Ner}(\mathcal{G}), \operatorname{Ner}(\operatorname{AUT}(\mathcal{K}))]
$$

where $\mathrm{AUT}(\mathcal{K})$ is the 2-groupoid with objects the groups $K_{x} \in \mathcal{K}$, 1-cells their group isomorphisms (regarded as functors), and 2-cells the natural transformations between them, and the square brackets mean homotopy classes of simplicial maps.

This theorem can be used, for example, to classify homotopy classes of continuous maps from a 1-type to a 2-type in terms of the said non abelian cohomology of groupoids.

\section{ACKnowledgments}

We wish to thank J. Duskin for his valuable comments. Our correspondence with him shows that most of our results were known to him and are implicitly contained in his work on nerves of bicategories. Even so we felt that our exposition may be useful for a large audience. We have used Duskin's clear geometric notation for the $n$-simplices and the corresponding convention for naming their faces. 


\section{REFERENCES}

[1] L. Breen. Théorie de Schereier supérieure. Ann. Sci. École Norm. Sup. (4), 25, 1992.

[2] M. Bullejos, E. Faro, and V. Blanco. Categorical non abelian cohomology and the Schreier theory of groupoids. http://www.ugr.es/ 'bullejos/schreier.pdf

[3] M. Bullejos, J. G. Cabello, and E. Faro. On the equivariant 2-type of a G-space. J. Pure Appl. Algebra, 129(3):215-245, 1998.

[4] M. Bullejos and A. M. Cegarra. On the geometry of 2-categories and their classifying spaces (extended version), http://www.ugr.es/^bullejos/geometryampl.pdf 2003.

[5] M. Bullejos and A. M. Cegarra. On the geometry of 2-categories and their classifying spaces. K-Theory, 29:211-229, 2003.

[6] J. M. Cordier. Sur les limites homotopiques de diagrammes homotopiquement cohérents. Compositio Math., 62:367-388, 1987.

[7] P. Dedecker. Les foncteurs $\operatorname{Ext}_{I I}, \mathrm{H}_{I I}^{2}$ et $\mathrm{H}_{I I}^{2}$ non abeliens. C. R. Acad. Sc. Paris, 258 Groupe 1:4891-4895, 1964.

[8] John W. Duskin. Simplicial matrices and the nerves of weak $n$-categories i: Nerves of bicategories. Theory and Applications of Categories, 9(10):198-308, 2002.

[9] J. R. Isbell. Adequate subcategories. Illinois J. Math., 4:541-552, 1960.

[10] J. R. Isbell. Subobjects, adequacy, completeness and categories of algebras. Razprawy Mat., 36:1-32, 1964

[11] A. Joyal and R. Street. Braided tensor categories. Advances in Math, 1(82), 1991.

[12] G. M. Kelly and R. Street. Review of the elements of 2-categories, volume 420 of Lecture Notes in Math. Springer-Verlag, 1974.

[13] Stephen Lack. A quillen model structure for 2-categories. K-Theory, 26(2):171-205, Jun 2002.

[14] I. Moerdijk and J. A. Svensson. The equivariant serre spectral sequence. Proc. Amer. Math. Soc., 118(1):263-277, 1993.

[15] Ieke Moerdijk and Jan-Alve Svensson. Algebraic classification of equivariant homotopy 2types, part i. J. of Pure and Applied Algebra, 89:187-216, 1993.

[16] O. Schreier. Uber die erweiterung von gruppen I. Monatshefte für Mathematik und Physik, 34:165-180, 1926

[17] O. Schreier. Uber die erweiterung von gruppen II. Abh. Math. Sem. Hamburg, 4:321-346, 1926.

[18] Ross Street. The algebra of oriented simplexes. J. of Pure and Applied Algebra, 49:283-335, 1987.

[19] Ross Street. Categorical structures, Handbook of Algebra Volume 1, pages 529-577. Elsevier Science, Amsterdam, 1996.

\author{
M. Bullejos, V. Blanco \\ Department of Algebra \\ University of Granada \\ 18071 Granada, Spain \\ bullejos@ugr.es \\ vblanco@ugr.es
}

E. Faro

Department of Appl. Mathematics

University of Vigo

36207 Vigo, Spain

efaro@dma.uvigo.es 